Accretion Phenomena and Related Outflows, IAU Colloquium 163

ASP Conference Series, Vol. 121, 1997

D.T. Wickramasinghe, L. Ferrario, and G.V. Bicknell, eds.

\title{
Stimulating Simulating
}

\author{
Rob Whitehurst
}

AMRL, 506 Lorimer Street, Vic. 3207, Australia

\begin{abstract}
The difficulties associated with numerical simulation of accretion discs are outlined along with a potential treatment, FLAME. This technique bridges the gap between existing finite difference codes and particle methods in modern computational fluid dynamics (CFD).
\end{abstract}

\section{Why current methods are inadequate.}

The direct numerical simulation of the complex phenomena associated with accretion processes is not a simple task. The difficulties associated with simulating even an ideal gas in the extreme environments present in astrophysical systems pushes the capabilities of modern CFD to its limits. The flows are usually hypersonic with free boundaries which must be included in the simulation. Furthermore both shear and shock waves are expected to be present and interacting with each other in most flow fields.

A good example of the above are the accretion discs of dwarf novae where there are both strong shear and shock waves. The shocks include that formed by the mass transfer stream as it impacts the disc and the spiral shocks predicted to lie within the disc proper. The interaction of these waves might be responsible for the angular momentum transfer in these discs. In addition their interplay with the system's tidal forces will be pivotal in the behaviour of both the thermal instability, (responsible for most dwarf novae outbursts), and the tidal instability, (i.e. the superhump phenomenon).

High quality simulations of accretion discs for comparison with observations and expectations would be very desirable. Unfortunately all existing simulations have shortcomings. With particle methods the high degree of diffussion and the unphysical interpenetration of flows degrade the results. In the case of accretion discs this is particularly problematic; the large velocity gradients due to the disc's shear are spread isotropically around each particle. Finite difference methods suffer from the constraint that the computed advection, work and impulses must always act with the grid. A shear wave that crosses the grid will be modelled as a 'staircase' which spawns many artificial shocks. Since the shear in a disc will not conform to an imposed grid this process is an effective generator of numerical dissipation and viscosity within the disc.

With even only the above dificulties it is perhaps not surprising that existing grid based codes and particle methods produce different descriptions of accretion discs. The fact that the models are crudely similar is encouraging, but insufficient detail is available to apply their results to the many outstanding problems in the field. 

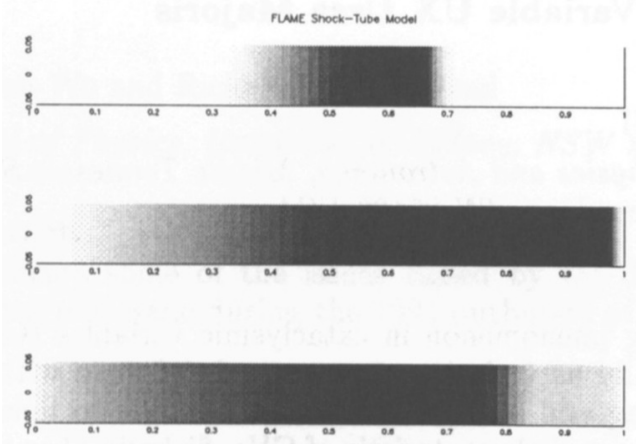

Figure 1. Three snapshots of a shock tube simulation.

\section{A Free Lagrange Method - FLAME}

To stimulate new progress a mesh based Lagrangian solver (FLAME) has been written which differs radically from both of the above methods. Like other grid based codes it contains (almost) no numerical viscosity, but since it is fully Lagrangian shear and shock waves can interact without the confounding influence of an imposed grid. FLAME is a development of the code described by Whitehurst (1995). It is identical in all important characteristics to that technique, but has been improved in generality, accuracy and ease of use. Although a Lagrangian technique which can handle flow fields through random meshes it also produces results of comparable quality to 'traditional' grid based codes. The figure is a grey scale plot of the gas velocity at three moments Sod (1978). In the first frame the shock is moving to the right with a rarefaction wave moving to the left. In the second frame the shock wave has just been reflected and in the last frame the reflected shock has passed through the contact front. The simulation was made using one thousand cells.

Currently FLAME can handle arbitrary two dimensional flow fields with reflecting, free flow, constrained flow or vacuum boundary conditions. The mesh can be initially regular or completely arbitrary, but the mesh will always ultimately conform to the flow. (The flow never conforms to the mesh in FLAME.) Work will soon commence on simulations of accretion discs.

\section{References}

Sod, G.A. 1978, J. Comp. Phys, 27, 1.

Whitehurst, R. 1995, MNRAS, 278, 356. 